\title{
Multi-residue analytical method for human pharmaceuticals and synthetic hormones in river water and sewage effluents by solid-phase extraction and liquid chromatography- tandem mass spectrometry.
}

\begin{abstract}
Pollutants such as human pharmaceuticals and synthetic hormones that are not covered by environmental legislation have increasingly become important emerging aquatic contaminants. This paper reports the development of a sensitive and selective multi-residue method for simultaneous determination and quantification of 23 pharmaceuticals and synthetic hormones from different therapeutic classes in water samples. Target pharmaceuticals include anti-diabetic, antihypertensive, hypolipidemic agents, $\beta 2$-adrenergic receptor agonist, antihistamine, analgesic and sex hormones. The developed method is based on solid phase extraction (SPE) followed by instrumental analysis using liquid chromatography-electrospray ionization-tandem mass spectrometry (LC-ESI-MS/MS) with 30 min total run time. River water samples $(150 \mathrm{~mL})$ and (sewage treatment plant) STP effluents $(100 \mathrm{~mL})$ adjusted to $\mathrm{pH} 2$, were loaded into MCX $(3 \mathrm{~cm} 3,60 \mathrm{mg})$ cartridge and eluted with four different reagents for maximum recovery. Quantification was achieved by using eight isotopically labeled internal standards (I.S.) that effectively correct for losses during sample preparation and matrix effects during LC-ESI-MS/MS analysis. Good recoveries higher than $70 \%$ were obtained for most of target analytes in all matrices. Method detection limit (MDL) ranged from 0.2 to $281 \mathrm{ng} / \mathrm{L}$. The developed method was applied to determine the levels of target analytes in various samples, including river water and STP effluents. Among the tested emerging pollutants, chlorothiazide was found at the highest level, with concentrations reaching up to $865 \mathrm{ng} / \mathrm{L}$ in STP effluent, and $182 \mathrm{ng} / \mathrm{L}$ in river water.
\end{abstract}

Keyword: Human pharmaceuticals; Synthetic hormones; Solid phase extraction (SPE); Liquid chromatography-tandem mass spectrometry (LC-MS/MS); Multi-residue analytical method; Aquatic pollution. 